\title{
Anatomía del Segmento Prehiliar de la Arteria Renal en Caninos
}

\author{
Anatomy of Prehiliar Segment of the Renal Artery in Dogs
}

\author{
Paulo Salinas,*;* \& Rodolfo Schwerter
}

\begin{abstract}
SALINAS, P. \& SCHWERTER, R. Anatomía del segmento prehiliar de la arteria renal en caninos. Int. J. Morphol., 33(3):1027-1031, 2015.

RESUMEN: La literatura anatómica describe, en general, que el riñón canino está irrigado por una arteria renal única. Estudios de campo han demostrado la existencia de variaciones anatómicas en el segmento prehiliar. Esta especie es utilizada como modelo urológico durante el entrenamiento quirúrgico por su fácil obtención, manipulación y tamaño. Estudios han descrito el patrón arterial intra-renal, sin embargo, la anatomía del segmento pre-hiliar de la arteria renal ha sido poco investigada. El objetivo del presente estudio fue describir el patrón arterial renal en el segmento pre-hiliar en caninos. Treinta pares de riñones de caninos fueron disectados y evaluados de acuerdo a 1) número de ramas por arterias renales únicas, 2) altura de origen de las arterias renales, 3) patrones de arteria renal doble y 4) lugar de bifurcación prehiliar de la arteria renal única. La aorta abdominal fue repletada con látex natural y posteriormente se disecó. Se observó arteria renal única y doble en el $90 \%$ y $10 \%$ de los casos, respectivamente. El número de ramas de la arteria renal única fue dos, tres y cuatro en el 50\%,33,3\% y 16,6\% de los casos, respectivamente. El origen de la arteria renal derecha se observó craneal, a igual altura y caudal a la arteria renal izquierda en el $80 \%, 13,3 \%$ y 6,6\% de los casos, respectivamente. En arterias renales dobles se observó un patrón únicaúnica y única-ramificado en el 33,3\%, 66,6\% de los casos, respectivamente. La bifurcación de la arteria renal única se observó en el tercio proximal, medio y distal en $8,33 \%, 29,16 \%$ y $62,50 \%$ de los casos, respectivamente. El estudio demostró que en la arteria renal y sus ramas no existe un patrón anatómico común descrito en las referencias, al contrario, existe una alta variabilidad anatómica.
\end{abstract}

PALABRAS CLAVE. Canino; Arteria renal; Variación anatómica; Segmento prehiliar.

\section{INTRODUCCIÓN}

El conocimiento anatómico de las arterias renales (a. renalis) es relevante en imagenología y en cirugía debido a la frecuencia del uso de riñones de animales en cirugía experimental. La exploración y tratamiento del trauma renal, procedimientos renales invasivos como nefrectomías y cirugías conservadoras, así como otras técnicas para manejar condiciones patológicas en el riñón han requerido la utilización de modelos animales durante el entrenamiento quirúrgico. El riñón de cerdo ha sido considerado el mejor modelo experimental urológico debido a su semejanza anatómica con el riñón humano, sin embargo, el alto costo de adquisición y mantenimiento, así como la gestión en laboratorio dificulta su utilización (Pereira-Sampaio et al., 2004). De este modo, en muchos países, el canino se utiliza como modelo urológico debido a su facilidad en la obtención, manipulación y tamaño adecuado. La literatura sobre el patrón arterial intrarrenal es amplia en diferentes especies, sin embargo, hasta ahora, la variación en la disposición de las estructuras renales prehiliares en caninos no ha generado interés. El objetivo del estudio fue la descripción anatómica de la morfología del segmento prehiliar de la arteria renal en caninos.

\section{MATERIAL Y MÉTODO}

El estudio fue de tipo descriptivo. Se utilizaron 60 riñones obtenidos de 30 caninos adultos (Canis lupus familiaris) de diferente sexo y razas, sometidos a eutanasia por enfermedades terminales de acuerdo a las normas establecidas por el Guide to the Care and Use of Experimental Animals (Olfert et al., 1998) o debido a muerte natural. Se utilizó la técnica anatómica de repleción vascular con látex y tinta china Rotring ${ }^{\circledR}$ a través de la aorta abdominal para observar las arterias renales de acuerdo a lo descrito previamente por Tompsett (1956). Posteriormente, los especímenes fueron mantenidos a $4{ }^{\circ} \mathrm{C}$ durante 2 días. Se realizó la disección desde el pedículo renal hacia el hilio.

* Laboratorio de Anatomía Veterinaria, Escuela de Medicina Veterinaria, Facultad de Recursos Naturales y Medicina Veterinaria, Universidad Santo Tomás, Temuco, Chile.

** Programa de Doctorado en Ciencias Morfológicas, Facultad de Medicina, Universidad de La Frontera, Temuco, Chile. 
Posteriormente las arterias renales fueron clasificadas de acuerdo a los siguientes criterios: 1) número de ramas por arterias renales únicas, 2) posición de bifurcación prehiliar de la arteria renal única, 3) altura de origen de las arterias renales y 4) patrones de arteria renal doble.

\section{RESULTADOS}

El 100\% de los riñones derechos y el $80 \%$ de los riñones izquierdos recibió una arteria renal. El 20\% de los riñones izquierdos presentó dos arterias renales, observándose estas solo en hembras (Figs. 1 y 2). En la Tabla I, se muestra el número y porcentaje total de ramas por arterias renales únicas. (Fig. 1A-C). Del total de arterias renales únicas observadas $(n=54)$ la posición de bifurcación en el segmento prehiliar fue proximal, medio y distal a la aorta en el $8,33 \%, 29,17 \%$ y $62,5 \%$, respectivamente (Tabla II; Fig. 1D-F). La altura de origen de las arterias renales en la aorta abdominal se presenta en la Tabla III (Fig. 1G-I). Del total de arterias renales dobles $(n=6)$ observadas en el estudio el 33,3\% presentó el patrón arterial "única - única" y el $66,6 \%$ presentó "única ramificada" (Fig. 2).

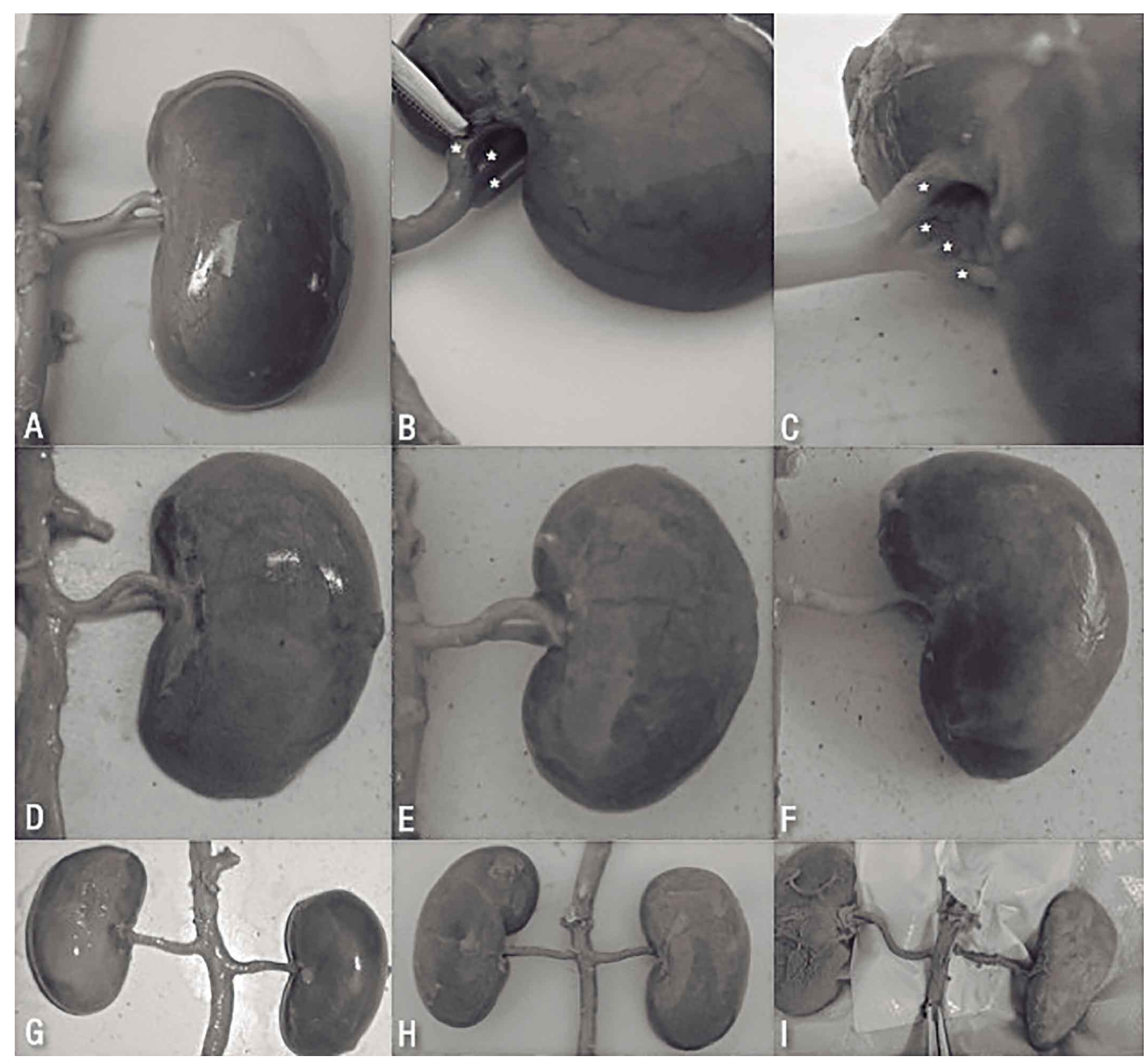

Fig. 1. Vista ventral de riñones caninos. Se observa en arteria renal única dos (A), tres (B) y cuatro (C) ramificaciones; bifurcación proximal (D), medio (E) y distal (F); y altura de origen cranial $(\mathrm{G})$, similar altura $(\mathrm{H})$ y caudal a la arteria renal izquierda (I) en la aorta abdominal. 


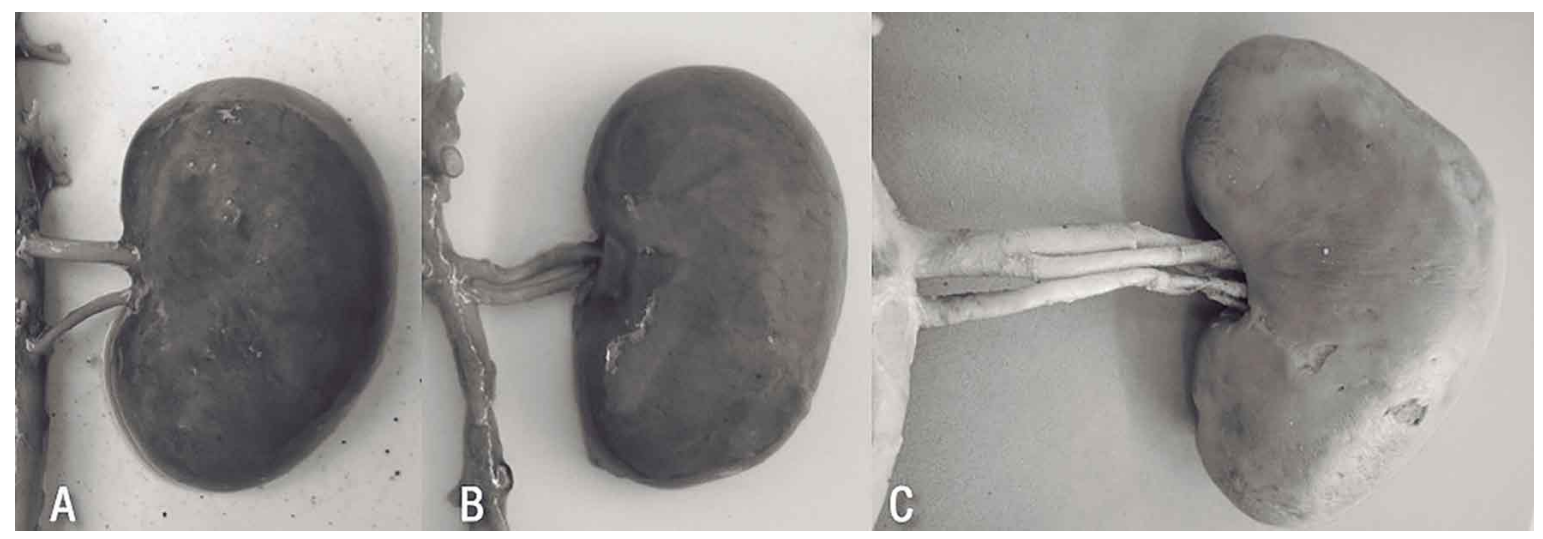

Fig. 2. Vista ventral de riñón canino izquierdo. Se observan dos patrones de arterias renales doble única - única (A, B), y patrón única - ramificada $(\mathrm{C})$.

Tabla I. Número de ramas por arterias renales únicas clasificadas por sexo y lado en caninos.

\begin{tabular}{llcccc}
\hline \multirow{2}{*}{ Sexo } & \multirow{2}{*}{ Lado } & \multicolumn{4}{c}{ Número de ramas } \\
\cline { 3 - 6 } & & I & II & III & IV \\
\hline \multirow{2}{*}{ Macho } & Derecho & 2 & 6 & 6 & --- \\
& Izquierdo & 4 & 6 & 2 & 2 \\
\multirow{2}{*}{ Hembra } & Derecho & 2 & 4 & 4 & 6 \\
\multirow{2}{*}{ Total } & Izquierdo & --- & 6 & 4 & --- \\
$(\%)$ & & 8 & 22 & 16 & 8 \\
\hline
\end{tabular}

Tabla II. Posición de bifurcación de la arteria renal única clasificada por sexo $(n=48)$.

\begin{tabular}{llccc}
\hline \multirow{2}{*}{ Sexo } & \multirow{2}{*}{ Lado } & \multicolumn{3}{c}{ Posición } \\
\cline { 3 - 5 } & & Proximal & Medio & Distal \\
\hline \multirow{2}{*}{ Macho } & Derecho & --- & 2 & 12 \\
& Izquierdo & --- & 4 & 8 \\
Hembra & Derecho & --- & 4 & 8 \\
& Izquierdo & 4 & 4 & 2 \\
Total & & 4 & 14 & 15 \\
$(\%)$ & & $(8,33)$ & $(29,16)$ & $(62,5)$ \\
\hline
\end{tabular}

Tabla III. Altura de origen de las arterias renales en la aorta.

\begin{tabular}{lcc}
\hline Origen & $\mathbf{n}$ & $\mathbf{\%}$ \\
\hline Arteria renal derecha cranial a la arteria renal izquierda. & 24 & 80 \\
Ambas arterias al mismo nivel. & 4 & 13,33 \\
Arteria renal derecha caudal a la arteria renal izquierda. & 2 & 6,66 \\
\hline
\end{tabular}

\section{DISCUSIÓN}

La anatomía del segmento prehiliar de las arterias renales en caninos no ha recibido mayor atención en los textos referenciales de anatomía animal. Sin embargo, durante una revisión de la literatura especializada en diferentes especies y disecciones previas realizadas en caninos demuestran la existencia de múltiples variaciones anatómicas en el segmento prehiliar tales como: arterias renales dobles y triples, con o sin ramificaciones, uni o bilaterales (Wiland \& Indykiewicz, 1999; Fernández Molina et al., 1996; Sajjarengpong \& Adirektaworn, 2006; Olave et al., 2007; Pestemalci et al., 2009; Trivedi et al., 2011; Shashikala et al., 2012; Daescu et al., 2012). Cabe señalar además, que la nomenclatura de las variaciones anatómicas reportadas en la arteria renal no está clara. Diferentes autores describen arterias adicionales, accesorias, hiliares o polares.
El entendimiento de las variaciones en el patrón pre-hiliar tiene su explicación en el desarrollo embrionario. Sampaio (2007) describió en humanos que el riñón inicialmente se ubica en la cavidad pélvica y se nutre de una extensa red de pequeños vasos aórticos denominados "red arterial urogenital" y gradualmente se desplaza hacia el espacio retroperitoneal en el abdomen. A medida que los riñones migran, vasos sanguíneos proximales suministran el aporte sanguíneo, sin embargo los vasos distales generalmente degeneran. Patasi \& Boozary (2009) mencionan que cada arteria renal única o múltiple está relacionada con arterias segmentares intra-renales, por lo que la presentación de arterias renales dobles o triples aumentaría el riesgo de hemorragia durante cirugías urológicas complejas, además de la posibilidad de isquemia segmentar e hipertensión postoperatoria. Por lo tanto, el conocimiento anatómico de las arterias múltiples es esencial previo a la realización de cualquier cirugía de trasplante, donde se empleen técnicas microvasculares para reconstruir arterias renales. 
En nuestro estudio las arterias renales dobles se originaron de la aorta abdominal e ingresaron al riñón a través del hilio. Además, la presentación de éstas fue similar a lo descrito por Marques-Sampaio et al. (2007) quienes reportaron en caninos arterias renales dobles y únicas en el 88,4\% y $11,6 \%$ de los casos, respectivamente. La presencia de arterias renales dobles (uni o bilaterales) se ha descrito en visones y caninos (Wiland \& Indykiewicz), humanos (Pereira-Sampaio et al., 2007), conejos (Nowicky et al., 2010) y cerdos (Sampaio et al., 1998). En caninos Shively (1978), Wiland \& Indykiewicz, Sajjarengpong \& Adirektaworn y Marques-Sampaio et al. reportaron la presencia de arterias renales dobles y señalaron que la mayor incidencia de presentación era en lado izquierdo y sólo en hembras, coincidiendo con nuestros resultados. Al contrario, Sampaio et al., describieron en cerdos una incidencia mayor en el lado derecho. Reportes embrionarios (Budhiraja et al., 2010; Evans, 1993; Gurses et al., 2009) describen que las arterias renales dobles se originan de ramas dorsales y ventrales que derivan directamente de la aorta abdominal y que el segmento prehiliar se bifurca en una rama dorsal y ventral. Patasi \& Boozary e Hyttel et al. (2010) describieron en un feto de $18 \mathrm{~mm}$ que el aporte sanguíneo al mesonefros, metanefros, glándulas suprarenales y gónadas era suministrado por nueve pares de arterias mesonéfricas laterales derivadas de la aorta abdominal, clasificadas como: grupo craneal (1er y 2do), intermedio (3ro al 5to) y caudal (6to a 9no). El grupo intermedio da lugar a las arterias renales, i.e. las arterias renales dobles son el resultado de la persistencia de una o mas arterias mesonéfricas laterales.

Es poco frecuente encontrar en la literatura científica la descripción de arterias renales triples en caninos (Shively). En humanos, Shashikala et al., describen diferencias en el calibre de las arterias renales dobles, y Bordei et al. (2004) le atribuyen un rol especialmente crítico e importante al vaso de mayor calibre sugiriendo un aporte predominante al territorio renal denominándolo: arteria renal principal y al vaso de menor calibre: arteria renal suplementaria. Los anatomistas y urólogos han demostrado que las ramas de la arteria renal no presentan anastomosis entre sí. Algunas de las ramas primarias y secundarias son denominadas arterias segmentares, las cuales irrigan los diferentes segmentos renales. Esto último sugiere que la segmentación del riñón canino se basa en el patrón de ramificación de la arteria renal (Pereira-Sampaio et al., 2004)

De acuerdo a Sajjarengpong \& Adirektaworn la cantidad de ramas terminales del segmento prehiliar la arteria renal en caninos pueden alcanzar hasta seis, sin embargo en nuestro estudio se observó un máximo de cuatro ramas, similar a lo reportado por Daescu et al., en humanos. Ambos estudios, coinciden en que dos ramas es el número más fre- cuente en ambas especies. Textos referenciales de anatomía veterinaria (Evans), describen de forma general, que el riñón canino está irrigado por una arteria renal; MarquesSampaio et al., mencionan además que ésta se bifurca en dos ramas, dorsal y ventral, y a partir de ramas primarias se originan ramas secundarias, cuyas ramificaciones (arterias interlobulares) se distribuyen en los polos craneal y caudal, y la región mesorenal.

Respecto a la altura de origen de las arterias renales, Evans y Smith (1999) señalaron que el origen de la arteria renal derecha es proximal a la arteria renal izquierda, sin embargo en nuestro estudio, observamos solo un caso en que la arteria renal izquierda se originaba distal a la arteria renal derecha, variante no descrita en la literatura consultada. Respecto a la posición de la bifurcación de la arteria renal, Sajjarengpong \& Adirektaworn y Marques-Sampaio et al., indicaron que la bifurcación se realiza en el tercio medio del segmento prehiliar, pero en nuestro estudio la posición más frecuente era distal. En relación a los patrones de arteria renal doble, en la investigación de Sajjarengpong \& Adirektaworn así como también en nuestro estudio, el patrón más frecuente fue "única - ramificada", seguido por el patrón "única - única”.

En conclusión, el nivel y patrón de ramificiación de la arteria renal en caninos se caracteriza por una alta variabilidad morfológica. En consecuencia, la anatomía del segmento prehiliar no debe ser considerada como un modelo constante, al contrario, los patrones anatómicos del segmento prehiliar son variables. Por lo tanto, sugerimos que la manipulación (clamping) de la arteria renal en canino es posible, pero dificil debido a la alta variabilidad morfológica e indiviual observada. En este estudio predominó el patrón única-única, lo que hace posible y seguro la utilización del riñón canino como modelo urológico quirúrgico en estudios experimentales en humano en los cuales la mofología de la arteria renal es un factor importante a considerar.

SALINAS, P. \& SCHWERTER, R. Anatomy of prehiliar segment of the renal artery in dogs. Int. J. Morphol., 33(3):1027-1031, 2015.

SUMMARY: The anatomical literature describes, in general, that the canine kidney is supplied by a single renal artery. Different studies demonstrate the existence of anatomical variations in the prehiliar segment. The dog is used as a model for its easy urological collection, handling and size, there are numerous studies of intrarenal arterial pattern, however, there have been few studies on the provision prehiliar segment of the renal artery. The study aimed to describe and standardize the renal artery in dogs. Thirty pairs of canine kidneys were dissected and evaluated according to number of arteries, branches and site of renal artery bifurcation. The abdominal aorta was repleted with natural rubber latex and 
subsequently dissected. Renal artery was observed and only two in $90 \%$ and $10 \%$ of cases, respectively. The number of branches of the renal artery was only two, three and four at $50 \%, 33.3 \%$ and $16.6 \%$ of cases, respectively. The origin of the right renal artery was observed cranial to the same head and flow to the left renal artery in $80 \%, 13.3 \%$ and $6.6 \%$ of cases, respectively. In double renal arteries a unique only-pattern and single-branched was observed in $33.3 \%, 66.6 \%$ of cases, respectively. The bifurcation of the renal artery was observed only in the proximal, middle and distal $8.33 \%, 29.16 \%$ and $62.50 \%$ of cases, respectively. The study showed that in the renal artery and its branches there is no common anatomical pattern described in the literature, there is however, a high anatomical variability.

KEY WORDS: Canine; Renal artery; Anatomical variation; Prehiliar segment.

\section{REFERENCIAS BIBLIOGRÁFICAS}

Bordei, P.; Sapte, E. \& Iliescu, D. Double renal arteries originating from the aorta. Surg. Radiol. Anat., 26(6):474-9, 2004.

Budhiraja, V.; Rastogi, R. \& Asthana, A. K. Renal artery variations: embryological basis and surgical correlation. Rom. J. Morphol. Embryol., 51(3):533-6, 2010.

Daescu, E.; Zahoi, D. E.; Motoc, A.; Alexa, A.; Baderca, F. \& Enache, A. Morphological variability of the renal artery branching pattern: a brief review and an anatomical study. Rom. J. Morphol. Embryol., 53(2):287-91, 2012.

Evans, E. H. Miller's Anatomy of The Dog. 3th ed. Toronto, W. B. Saunders Company, 1993.

Fernández Molina, J. C.; Lecuona, E.; Gallego, E.; García Pérez, J. J. \& Martín Vasallo, P. Desarrollo renal: Factores de crecimiento, celularidad y transporte de sodio y potasio. Nefrol., 16(1):26-37, 1996.

Gurses, I. A.; Kale, A.; Gayretli, O.; Bayraktar, B.; Usta, A.; Kayaalp, M. \& Ari Z. Bilateral variations of renal and testicular arteries. Int. J. Anat. Var., 2:45-7, 2009.

Hyttel, P.; Sinowatz, F.; Vejlsted, M. \& Betteridge, K. Essentials of Domestic Animal Embriology. Philadelphia, Saunders Elsevier, 2010.

Marques-Sampaio, B. P.; Pereira-Sampaio, M. A.; Henry, R. W.; Favorito, L. A. \& Sampaio, F. J. Dog kidney: anatomical relationships between intrarenal arteries and kidney collecting system. Anat. Rec. (Hoboken), 290(8):1017-22, 2007.

Nowicki, W.; Brudnicki, W.; Iwan'czyk, M.; Jab?on’ski, R. \& Skoczylas, B. Variation in branches of the abdominal aorta in european rabbit. Electron. J. Pol. Agric. Univ., 13(4):10, 2010.

Olave, E.; Henríquez, J.; Cruzat, C.; Puelma, F. \& Soto, A. Arterias renales múltiples. Int. J. Morphol., 25(4):927-30, 2007.
Olfert, E. D.; Cross, B. M. \& McWilliam, A. A. Guide to the Care and Use of Experimental Animals. Ontario, Canadian Council on Animal Care, 1998.

Patasi, B. \& Boozary, A. A case report: accessory right renal artery. Int. J. Anat. Var., 2:119-21, 2009.

Pereira-Sampaio, M. A.; Favorito, L. A. \& Sampaio, F. J. Pig kidney: anatomical relationships between the intrarenal arteries and the kidney collecting system. Applied study for urological research and surgical training. J. Urol., 172(5 Pt. 1):2077-81, 2004.

Pereira-Sampaio, M.; Favorito, L. A.; Henry, R. \& Sampaio, F. J. Proportional analysis of pig kidney arterial segments: differences from the human kidney. J. Endourol., 21(7):784-8, 2007.

Pestemalci, T.; Mavi, A.; Yildiz, Y. Z.; Yildirim, M. \& Gumusburun, E. Bilateral triple renal arteries. Saudi J. Kidney Dis. Transpl., 20(3):468-70, 2009.

Sajjarengpong, K. \& Adirektaworn, A. The variations and patterns of renal arteries in dogs. Thai J. Vet. Med., 36(1):39-46, 2006.

Sampaio, F. J. B. Anatomia Renal para Urologia. Rio de Janeiro, Gráfica e Editora Prensa, 2007.

Sampaio, F. J.; Pereira-Sampaio, M. A. \& Favorito, L. A. The pig kidney as an endourologic model: anatomic contribution. J. Endourol., 12(1):45-50, 1998.

Shashikala, P.; Anjali, W.; Anshuman, N. \& Jayshree, D. A case report: double renal arteries. Int. J. Anat. Var., 5:22-4, 2012.

Shively, M. J. Origin and branching of renal arteries in the dog. J. Am. Vet. Med. Assoc., 173(8):986-9, 1978.

Smith, B. J. Canine Anatomy. Philadelphia, Lippincott Williams \& Wilkins, 1999.

Tompsett, D. H. Anatomical Techniques. Edinburgh, E. \& S. Livingstone, 1956.

Trivedi, S.; Athavale, S. \& Kotgiriwar, S. Normal and variant anatomy of renal hilar structures and its clinical significance. Int. J. Morphol., 29(4):1379-83, 2011.

Wiland, C. \& Indykiewicz, P. Multiple renal arteries (aa. renales) in mink and dog. Electron. J. Pol. Agric. Univ., 2(2):03, 1999.

Dirección para Correspondencia:

Paulo C. Salinas Pérez, MV, MSc.

Laboratorio de Anatomía Veterinaria

Escuela de Medicina Veterinaria

Universidad Santo Tomás

Manuel Rodríguez 060, Temuco

CHILE

Email: psalinas@santotomas.cl

Recibido: 29-07-2014

Aceptado: 01-06-2015 